\title{
CONSECUTIVE COVARIANT CONFIGURATIONS AT A POINT OF A SPACE CURVE*
}

BY

\section{ABBA V. NEWTON \\ I. INTRODUCTION}

A systematic study of the projective differential geometry of space curves was first made by Halphen $\dagger$ in a memoir of 1880 . Wilczynski $\ddagger$ in 1905 and 1906 and Sannia $\S 1926$ made important additions to the subject.

The projective differential theory of a curve involves many configurations associated covariantly with the curve. The purpose of this paper is to make some contributions to the theory of a space curve, which are based upon the study of consecutive configurations. The work follows the lines of a similar investigation made by Lane $\|$ for the case of a plane curve.

We now make precise the meaning of the word "consecutive" as used in the present paper. Let us consider an analytic curve $C$ in projective space of three dimensions. The equations of such a curve, in non-homogeneous projective coordinates $x, y, z$, can be written in the form of two power series expansions,

$$
y=a_{0}+a_{1} x+a_{2} x^{2}+\cdots, \quad z=c_{0}+c_{1} x+c_{2} x^{2}+\cdots,
$$

which represent $C$ in the neighborhood of the ordinary point $P$ with coordinates $0, a_{0}, c_{0}$; the neighborhood is supposed to be sufficiently small so that the series converge. If $Q$ is a point with coordinates $h, k, l$ on $C$ near $P$, then $h, k, l$ must satisfy the foregoing equations when substituted in place of $x$, $y, z$, respectively:

$$
k=a_{0}+a_{1} h+a_{2} h^{2}+\cdots, l=c_{0}+c_{1} h+c_{2} h^{2}+\cdots .
$$

If, now, $h$ is regarded as an infinitesimal, and if we are considering problems

* Presented to the Society, June 22, 1933; received by the editors June 24, 1933.

† G. H. Halphen, Sur les invariants differentiels des courbes gauches, Journal de l'Ecole Polytechnique, vol. 28 (1880), p. 1. p. 99.

$\ddagger$ E. J. Wilczynski, General projective theory of space curves, these Transactions, vol. 6 (1905), 1906.

E. J. Wilczynski, Projective Differential Geometry of Curves and Ruled Surfaces, B. G. Teubner,

\$. Sannia, Nuova trattazione della geometria proiettivo-differenziale delle curve sghembe (memoria 2), Annali di Matematica, (4), vol. 3 (1926), p. 1.

II E. P. Lane, On the projective differential geometry of plane curves, Tôhoku Mathematical Journal, vol. 37 (1933), p. 423. 
in which the powers of $h$ higher than the first are negligible in comparison with the first power, we can drop all terms after the second in each series and write

$$
k=a_{0}+a_{1} h, \quad l=c_{0}+c_{1} h .
$$

In such a case the point $Q$ is said to be "consecutive" to the point $P$. Moreover, the tangent line of the curve at $Q$ is said to be "consecutive" to the tangent line at $P$, and similarly for other corresponding covariant configurations associated with the points $P$ and $Q$.

For the development of the theory of consecutive configurations we need first to call to mind some fundamental facts from the projective differential geometry of a space curve. This is done in §II. In this section are described certain configurations covariantly associated with a point $P$ of a space curve. These determine geometrically the vertices and unit point of a local coordinate system at the point $P$. Equations (1), when referred to this local coordinate system, reduce to the canonical form with which the section opens.

In §III we consider the configurations associated with a point $Q$ consecutive to the point $P$ as those of $\S I I$ are associated with $P$. Their equations referred to the local coordinate system at the point $P$ are determined. From these can be found the coordinates, in the same coordinate system at $P$, of the vertices and unit point of the local coordinate system associated with the point $Q$, that is, the consecutive local coordinate system. This makes it possible to set up the equations of transformation between the two local coordinate systems as is also done in the section. Lastly, the equations of the curve $C$ referred to the consecutive coordinate system are deduced.

The application of this theory is made in $\S I V$. There we consider three main types of problems. All of these have in common, however, the feature that the point or curve to be determined depends upon two consecutive curves, or upon two consecutive points or surfaces, as the case may be.

\section{Fundamentals OF SPACE CURVE THEORY}

In the theory of consecutive covariant configurations associated with a space curve, we restrict ourselves, as we have already stated, to the case of an analytic curve $C$. It is provable in projective differential geometry that for such a curve equations (1), when referred to a particular covariant local coordinate system, assume the simple form

$$
\begin{aligned}
& y=x^{2}+a x^{7}+b x^{8}+e x^{9}+\cdots, \\
& z=x^{3}+x^{6}+c x^{7}+d x^{8}+g x^{9}+\cdots .
\end{aligned}
$$

It is the purpose of this section to recall to the reader's mind the facts which 
give geometric significance to the vertices of the tetrahedron of reference and to the unit point of the particular coordinate system involved.*

We consider on the curve $C$ the point $P$ which is the vertex $(0,0,0)$ of the tetrahedron of reference. The tangent line to $C$ at $P$ has the equations $y=z=0$, and the osculating plane at $P$ has the equation $z=0$. The equations of the osculating twisted cubic at the point $P$, that is, the twisted cubic having six-point contact with the curve $C$ at $P$, has the equations

$$
y=x^{2}, \quad z=x^{3},
$$

and its osculating conic at $P$, which by definition is the osculating conic of $C$ at $P$, is given in homogeneous form by the equations

$$
4 x_{1} x_{3}-3 x_{2}^{2}=x_{4}=0,
$$

where

$$
x_{2} / x_{1}=x, \quad x_{3} / x_{1}=y, \quad x_{4} / x_{1}=z .
$$

The null system of the osculating cubic has the equations

$$
\xi_{1}=y_{4}, \quad \xi_{2}=-3 y_{3}, \quad \xi_{3}=3 y_{2}, \quad \xi_{4}=-y_{1} .
$$

The bundle of quadric surfaces having seven-point contact with the curve $C$ at the point $P$ is represented by the equation

$$
\alpha\left(y-x^{2}\right)+\beta\left(y^{2}-z x\right)+\gamma\left(z-x y-z^{2}\right)=0,
$$

in which the coefficients $\alpha, \beta, \gamma$ are arbitrary constants. These quadrics have as an eighth point of intersection, the point of Sannia, which, in homogeneous coordinates, is the point $(1,0,0,1)$. In this bundle of quadrics there is one cone, called the osculating quadric cone, whose vertex is at the point $P$. Its equation is

$$
y^{2}-z x=0 .
$$

All the quadric surfaces which pass through the osculating cubic of the curve $C$ at the point $P$ form another bundle with the equation

$$
\alpha\left(y-x^{2}\right)+\beta\left(y^{2}-z x\right)+\gamma(z-x y)=0,
$$

in which again $\alpha, \beta, \gamma$ are quite arbitrary. Two cones of this bundle are also seven-point cones. One of these is the osculating quadric cone; the other has the equation

$$
y-x^{2}=0
$$

* For a convenient reference for the derivation of the results mentioned in this section (with the exception of the surface of Calapso) see E. P. Lane, Projective Differential Geometry of Curves and Surfaces, University of Chicago Press, 1932, pp. 20-25. 
its vertex is at the point $(0,0,0,1)$ called the Halphen point corresponding to the point $P$ of the curve $C$.

We next recall the definitions of the principal plane and of the principal point of the tangent. Let the curve $C$ and its osculating cubic at the point $P$ be projected onto their common osculating plane $z=0$ from a point not on that plane. In general, the projections have six-point contact. If, however, the center of projection lies in the plane $y=0$, they have seven-point contact. This plane $y=0$ is called the principal plane at the point $P$ of the curve $C$. The point $(0,1,0,0)$, which corresponds to the principal plane in the null system of the osculating cubic given by equations (5), is the principal point of the tangent line at $P$.

The polar line of this principal point with respect to the osculating conic of the curve $C$ at the point $P$ meets the osculating conic in $P$ and in the point $(0,0,1,0)$. This point together with the principal point and the point of Sannia $(1,0,0,1)$ determines the plane $x_{1}-x_{4}=0$, one of whose three intersections with the osculating cubic is the unit point $(1,1,1,1)$.

Lastly, a configuration associated with a point $P$ of the curve $C$, but not assisting in the characterization of our coordinate system, is the surface of Calapso.* It is the locus of the vertices of the six-point quadric cones at $P$ of the curve $C$. Its equation, in homogeneous coordinates since that is the form in which we shall make use of it, is known to be

$$
x_{1} x_{4}^{2}-3 x_{2} x_{3} x_{4}+2 x_{3}^{8}=0 .
$$

This surface is, in fact, a cubic ruled surface of the Cayley type, sometimes called a Cayley cubic scroll.

\section{TRANSFORMation OF CONSECUTIVE LOCAL COORDINATES}

With every point on the curve $C$ there may be associated a coordinate system which is related to the point in the same geometric manner as the coordinate system just described is related to the point $P$. In particular, there is such a coordinate system, which we shall refer to as the consecutive coordinate system, associated with the point $Q$ consecutive to $P$. In this section we wish to find the equations of transformation between the original coordinate system at $P$ and the consecutive one at $Q$.

To accomplish our purpose, we make use of a suitable auxiliary transformation of the coordinates $x, y, z$ into new coordinates $\xi, \eta, \zeta$ in an auxiliary coordinate system having the point $Q$ as origin $(0,0,0)$. When the equations of the curve $C$ have been transformed to these new coordinates, we are ready

* R. Calapso, Sulle superficie gobbe di terzo grado (del tipo di Cayley) legate al punto di una data superficie, Rendiconti dei Lincei, (6), vol. 13 (1931), p. 495. 
to obtain in the coordinates $\xi, \eta, \zeta$ the equations of the configurations related to the point $Q$ as those of the preceding section are related to the point $P$. The inverse of the auxiliary transformation already used now permits us to transform these equations back to the original coordinate system, and hence we can find the coordinates in the $x y z$-system of the vertices of the consecutive tetrahedron of reference and of the consecutive unit point. Since five points, no four of which are coplanar, whose coordinates in each of two systems are known, are sufficient to determine the transformation between the two systems, the transformation between the original and the consecutive coordinate systems can now be established.

We introduce first the auxiliary transformation and then apply it to the equations of the curve $C$. Let us impose on the new coordinate system, besides the condition that the point $Q$ consecutive to the point $P$ be the origin $(0,0,0)$, the further conditions that the line $\eta=\zeta=0$ be the tangent to $C$ at $Q$, and that the plane $\zeta=0$ be the osculating plane. We consider what these conditions imply for the coordinates $x, y, z$. For a point with coordinates $h, k, l$ near the point $P$ on the curve $C$, equations (2) tell us that

$$
\begin{aligned}
k & =h^{2}+a h^{7}+b h^{8}+e h^{9}+\cdots, \\
l & =h^{3}+h^{6}+c h^{7}+d h^{8}+g h^{9}+\cdots .
\end{aligned}
$$

If, now, the point is $Q$, the point consecutive to $P$ on $C$, so that we can neglect powers of $h$ higher than the first, the coordinates $x, y, z$ of $Q$ become $h, 0,0$. The general equations of the tangent to $C$ at a point $(\bar{x}, \bar{y}, \bar{z})$ are

$$
y-\bar{y}-(x-\bar{x}) \bar{y}^{\prime}=0, \quad z-\bar{z}-(x-\bar{x}) \bar{z}^{\prime}=0,
$$

where $\bar{y}^{\prime}$ and $\bar{z}^{\prime}$ mean $d y / d x$ and $d z / d x$, respectively, evaluated at the point $(\bar{x}, \bar{y}, \bar{z})$. Hence, the equations of the tangent to $C$ at $Q(h, 0,0)$ are

$$
y-2 h x=z=0,
$$

since we neglect powers of $h$ higher than the first. The general equation of the osculating plane to $C$ at a point $(\bar{x}, \bar{y}, \bar{z})$ can be written in the form

$$
z-\bar{z}-(x-\bar{x}) \bar{z}^{\prime}-\frac{\left[y-\bar{y}-(x-\bar{x}) \bar{y}^{\prime}\right] \bar{z}^{\prime \prime}}{\bar{y}^{\prime \prime}}=0 .
$$

For the point $Q$, this becomes

$$
z-3 h y=0 .
$$

Summarizing results, we find that the point $(h, 0,0)$ in the original coordinate system becomes the point $(0,0,0)$ in the new; the line $y-2 h x=z=0$ in the old, becomes the line $\eta=\zeta=0$; and the plane $z-3 h y=0$ becomes the plane $\zeta=0$. The 
auxiliary transformation is completely determined by these relationships; its equations are

$$
\xi=x-h, \quad \eta=y-2 h x, \quad \zeta=z-3 h y,
$$

or, in homogeneous form,

(10) $\quad \sigma \xi_{1}=x_{1}, \quad \sigma \xi_{2}=x_{2}-h x_{1}, \quad \sigma \xi_{3}=x_{3}-2 h x_{2}, \quad \sigma \xi_{4}=x_{4}-3 h x_{3}$,

where $\sigma$ is a proportionality factor. The inverse of the non-homogeneous form is

$$
x=\xi+h, \quad y=2 h \xi+\eta, \quad z=3 h \eta+\zeta .
$$

When equations (2) are subjected to transformation (11), we obtain the equations of $C$ referred to the new coordinate system, namely,

$$
\begin{aligned}
& \eta=\xi^{2}+7 a h \xi^{6}+(a+8 b h) \xi^{7}+\cdots, \\
& \zeta=\xi^{3}+6 h \xi^{5}+(1+7 c h) \xi^{6}+[c+h(8 d-3 a)] \xi^{7}+\cdots
\end{aligned}
$$

Our next problem is to determine the covariant configurations at the point $Q$, by means of which the vertices of the consecutive tetrahedron of reference and the consecutive unit point may be characterized. We first consider the consecutive osculating cubic. Its parametric equations in homogeneous coordinates are

$$
\xi_{1}=1+12 h t^{2}, \quad \xi_{2}=t+6 h t^{3}, \quad \xi_{3}=t^{2}, \quad \xi_{4}=t^{3} .
$$

We shall verify this by showing that these equations satisfy the equations (12) of the curve $C$ through terms in $\xi^{5}$. By setting

$$
\xi=\xi_{2} / \xi_{1}, \quad \eta=\xi_{3} / \xi_{1}, \quad \zeta=\xi_{4} / \xi_{1},
$$

we obtain the non-homogeneous form of equations (13), namely,

$$
\xi=t-6 h t^{3}, \quad \eta=t^{2}-12 h t^{4}, \quad \zeta=t^{3}-12 h t^{5} .
$$

If we invert the first of these we get

$$
t=\xi+6 h \xi^{3} .
$$

When this expression for $t$ is substituted in the last two of equations (14), we have the non-homogeneous equations of the osculating cubic:

$$
\eta=\xi^{2}, \quad \zeta=\xi^{3}+6 h \xi^{5},
$$

which obviously coincide with equations (12) through terms in $\xi^{5}$. Referred to the original coordinate system we may write the equations of the consecutive osculating cubic in the form

$$
y=x^{2}, \quad z=x^{3}+6 h x^{5} .
$$


To introduce the consecutive osculating conic, let us first consider the tangent line to the cubic at a point $\xi$. It intersects the osculating plane $\xi_{4}=0$ of the cubic at the point $Q$ in one point. The locus of this point of intersection as the point $\xi$ varies over the cubic is by definition the osculating conic of the cubic at the point $Q$; it is also called the osculating conic of the curve $C$ at the point $Q$, or the consecutive osculating conic. Since the tangent line is determined by the points $\xi$ and $\xi^{\prime}$ whose coordinates are given respectively by equations (13) and by the equations obtained by differentiating (13) with respect to $t$, its parametric equations are

$$
\begin{array}{ll}
\xi_{1}=1+12 h t^{2}+24 h \lambda t, & \xi_{2}=t+6 h t^{3}+\lambda\left(1+18 h t^{2}\right), \\
\xi_{3}=t^{2}+2 \lambda t, & \xi_{4}=t^{3}+3 \lambda t^{2} .
\end{array}
$$

It meets the plane $\xi_{4}=0$ in the point with coordinates

$$
\xi_{1}=1+4 h t^{2}, \quad \xi_{2}=2 t / 3, \quad \xi_{3}=t^{2} / 3, \quad \xi_{4}=0 .
$$

The locus of this point as $\xi$ varies along the cubic is found, by eliminating $t$ from these equations, to be

$$
4 \xi_{1} \xi_{3}-48 h \xi_{3}^{2}-3 \xi_{2}^{2}=\xi_{4}=0 .
$$

Making use of equations (10), we obtain the equation of the consecutive osculating conic referred to the original coordinate system:

$$
4 x_{1} x_{3}-2 h x_{1} x_{2}-48 h x_{3}^{2}-3 x_{2}^{2}=x_{4}-3 h x_{3}=0 .
$$

We shall next interest ourselves in the determination of the bundle of quadric surfaces having seven-point contact with the curve $C$ at the point $Q$ and of their eighth point of intersection, which is the consecutive point of Sannia. Let us write the general equation of the second degree in $\xi, \eta, \zeta$ and impose the condition that it be satisfied identically in $\xi$, through terms of the sixth degree, by the power series (12) for $\eta$ and $\zeta$. This gives for a general one of the seven-point quadrics, the equation

$$
\begin{aligned}
& \alpha\left(\eta-\xi^{2}-7 a h \zeta^{2}\right)+\beta\left(\eta^{2}-\xi \zeta+6 h \zeta^{2}\right) \\
& \quad+\gamma\left[\zeta^{2}+\xi \eta-\zeta+h\left(7 c \zeta^{2}+6 \eta \zeta\right)\right]=0,
\end{aligned}
$$

in which $\alpha, \beta, \gamma$ are arbitrary constants. After making use of transformation (9) we have the result that the equation of the bundle of seven-point quadrics referred to the original coordinate system is

$$
\begin{aligned}
& \alpha\left(y-x^{2}-7 a h z^{2}\right)+\beta\left[y^{2}-x z+h\left(z+6 z^{2}-x y\right)\right] \\
& \quad+\gamma\left[x y+z^{2}-z+h\left(7 c z^{2}+6 y z-4 y-2 x^{2}\right)\right]=0 .
\end{aligned}
$$

The eighth point of intersection of all the quadrics of this bundle can be deter- 
mined as the eighth intersection point of the three particular seven-point quadrics whose equations are, respectively,

$$
\begin{aligned}
y-x^{2}-7 a h z^{2} & =0, \\
y^{2}-x z+h\left(z+6 z^{2}-x y\right) & =0, \\
x y+z^{2}-z+h\left(7 c z^{2}+6 y z-4 y-2 x^{2}\right) & =0 .
\end{aligned}
$$

It is easy to solve these equations if we notice that, since for $h=0$ the eighth solution must be $(0,0,1)$, we can suppose that our required solution will be of the form ( $h m, h n, 1+h r)$. When we substitute these expressions for $x, y, z$ in equations (18) and neglect powers of $h$ higher than the first, we obtain simple equations which readily give $(7 h, 7 a h, 1-7 c h)$ as the eighth solution of equations (18). In homogeneous coordinates, then, the consecutive point of Sannia referred to the original coordinate system is the point

$$
(1,7 h, 7 a h, 1-7 c h) \text {. }
$$

Among the seven-point quadrics there is one cone having its vertex at the point $Q$. It is found by making equation (17) homogeneous and imposing the condition that the four first partial derivatives of the left member be zero at the point $(1,0,0,0)$. In this way we get the conditions $\alpha=\gamma=0$. When these values are substituted in equation (17), the equation

$$
\eta^{2}-\xi \zeta+6 h \zeta^{2}=0
$$

is obtained. Hence, the equation of the osculating quadric cone referred to the original coordinate system is

$$
y^{2}-x z+h\left(z+6 z^{2}-x y\right)=0 .
$$

We next consider the bundle of quadrics through the consecutive osculating cubic, and determine in it the cone different from the osculating quadric cone which is also a seven-point cone. Its vertex will be the consecutive Halphen point. We write again the general equation in $\xi, \eta, \zeta$ of a quadric surface. This time we demand that it be identically satisfied in $t$ by equations (14) of the consecutive osculating cubic. The result is

$$
\alpha\left(\eta-\xi^{2}\right)+\beta\left(\eta^{2}-\xi \zeta+6 h \zeta^{2}\right)+\gamma(\xi \eta-\zeta+6 h \eta \zeta)=0,
$$

wherein $\alpha, \beta, \gamma$ are arbitrary constants. To find the condition that one of these quadrics be also a seven-point quadric, we compare the left members of this equation and of equation (17), thus obtaining the relation

$$
\gamma=7 a h \alpha
$$

between the arbitrary constants. If, moreover, this quadric is to be a cone, 
we have a further relation

$$
\alpha \beta=-12 \alpha^{2} h,
$$

obtained by setting the discriminant of (20) equal to zero and simplifying by means of the first relation. If $\alpha=0$, then $\gamma=0$ and the cone is the osculating quadric cone, with which we are not at the moment concerned. Hence, for our desired cone we know that $\beta=-12 \alpha h$, since $\alpha \neq 0$. Therefore, the equation of the cone is

$$
\eta-\xi^{2}+12 h\left(\xi \zeta-\eta^{2}\right)+7 a h(\xi \eta-\zeta)=0,
$$

or, in the coordinates $x, y, z$,

$$
y-x^{2}+12 h\left(x z-y^{2}\right)+7 a h(x y-z)=0 .
$$

To find the coordinates of its vertex we write this last equation in homogeneous form and set the four first partial derivatives of the left member equal to zero. The solution $(0,6 h, 7 a h, 1)$ of the four equations so obtained is the vertex of the cone or the consecutive Halphen point. Summarizing, we can state that the seven-point cone through the consecutive osculating cubic, which is not the osculating quadric cone, is given in the original coordinates by equation (21). Its vertex, the consecutive Halphen point, has the coordinates

$$
0,6 h, 7 a h, 1 \text {. }
$$

Our next concern will be with the consecutive principal plane. To find it we first determine the equations of the projections of the curve $C$ and of the consecutive osculating cubic onto the osculating plane $\zeta=0$, the center of projection being a general point not on the plane. Then we find the condition that these projected curves have seven-point contact. The equations of a line joining the point $(\bar{\xi}, \bar{\eta}, \bar{\zeta})$ of $C$ and any point $(\alpha, \beta, \gamma)$ not in the plane $\zeta=0$ are

$$
\begin{aligned}
& \xi=\alpha+(\bar{\xi}-\alpha) \rho, \\
& \eta=\beta+(\bar{\eta}-\beta) \rho, \\
& \zeta=\gamma+(\bar{\zeta}-\gamma) \rho,
\end{aligned}
$$

where $\rho$ is a parameter. By setting $\rho=-\gamma /(\bar{\zeta}-\gamma)$ the equations of the projection of the curve $C$ from the point $(\alpha, \beta, \gamma)$ onto the plane $\zeta=0$ are found to be

$$
\xi=\alpha-\gamma(\bar{\xi}-\alpha) /(\bar{\zeta}-\gamma), \quad \eta=\beta-\gamma(\bar{\eta}-\beta) /(\bar{\zeta}-\gamma) .
$$

Upon expanding the right members of these equations and replacing $\bar{\eta}$ and $\bar{\zeta}$ by their values in terms of $\bar{\xi}$ given by equations (12) of the curve $C$, we 
obtain the form

$$
\begin{aligned}
& \xi=\bar{\xi}-\alpha \bar{\xi}^{8} / \gamma+\bar{\xi}^{4} / \gamma-6 h \alpha \bar{\xi}^{5} / \gamma-[\alpha+\gamma(\alpha+7 \alpha c h-6 h)] \bar{\xi}^{8} / \gamma^{2}-\cdots \\
& \eta=\bar{\xi}^{2}-\beta \bar{\xi}^{8} / \gamma-(6 h \beta-1) \overline{\xi^{5}} / \gamma-\left[\beta+\beta \gamma(1+7 c h)-7 a h \gamma^{2}\right] \overline{\xi^{6}} / \gamma^{2}-\cdots
\end{aligned}
$$

When the first of these is inverted and the power series for $\bar{\xi}$ in terms of $\xi$ so obtained is substituted for $\bar{\xi}$ in the second, the resulting equation of the projection of $C$ onto the osculating plane $\zeta=0$ is

$$
\begin{aligned}
\eta= & \xi^{2}-\beta \xi^{3} / \gamma+2 \alpha \xi^{4} / \gamma-[(1+6 h \beta) \gamma+3 \alpha \beta] \xi^{\xi} / \gamma^{2} \\
& +\left[7 \alpha^{2}+2 \beta-\beta \gamma+h\left(7 a \gamma^{2}+12 \alpha \gamma-7 c \beta \gamma\right)\right] \xi^{6} / \gamma^{2}+\cdots .
\end{aligned}
$$

By a similar procedure, the projection of the osculating cubic onto the plane $\zeta=0$ is found. It proves to be the same as the projection of $C$ through terms of the sixth degree in $\xi$, except that in the coefficient of $\xi^{6}$ the terms

$$
\left[-\beta \gamma+h\left(7 a \gamma^{2}-7 c \beta \gamma\right)\right] / \gamma^{2}
$$

are missing. Hence, if the two projections are to have seven-point contact, this expression must vanish. This means that

$$
\beta+7 \operatorname{ch} \beta-7 a h \gamma
$$

must equal zero, for we know $\gamma \neq 0$ since the center of projection does not lie in the plane $\zeta=0$. This relation between the coordinates $\beta$ and $\gamma$ merely implies that the center of projection must lie in the plane with the equation

$$
(1+7 c h) \eta-7 a h \zeta=0 .
$$

This plane is by definition the consecutive principal plane. After applying transformation (9), we have the equation of the consecutive principal plane in the coordinates $x, y, z$, namely,

$$
2 h x-(1+7 c h) y+7 a h z=0 .
$$

As the consecutive principal point is the point corresponding to the consecutive principal plane in the null system of the consecutive osculating cubic, it will now be necessary to determine this null system. The osculating plane at any point $\xi$ of the cubic is found, by differentiating (13) twice and writing the equation of the plane determined by $\xi, \xi^{\prime}, \xi^{\prime \prime}$, to be

$$
t^{3} \xi_{1}-3 t^{2} \xi_{2}+\left(3 t-12 h t^{3}\right) \xi_{3}-\left(1-18 h t^{2}\right) \xi_{4}=0,
$$

where $t$ is the value of the parameter corresponding to the particular $\xi$ chosen. Since this is a cubic equation in $t$ there are in general three values of $t$ which will satisfy it for any arbitrarily chosen values $\xi_{1}, \xi_{2}, \xi_{3}, \xi_{4}$. Therefore, through 
any point $\eta$ of $S_{3}$ there pass three osculating planes of the osculating cubic. If we let $t_{1}, t_{2}, t_{3}$ be the parametric values corresponding to the three points of osculation, we can easily write the equation of the plane determined by these points. When it is simplified by means of the values obtained from equation (22) of the elementary symmetric functions of $t_{1}, t_{2}, t_{3}$, it takes the form

$$
\eta_{4} \xi_{1}-3 \eta_{3} \xi_{2}+\left(3 \eta_{2}-30 h \eta_{4}\right) \xi_{3}-\left(\eta_{1}-30 h \eta_{3}\right) \xi_{4}=0 .
$$

By applying transformation (10) we reach the corresponding equation in the old coordinates, namely,

$$
y_{4} x_{1}-3 y_{3} x_{2}+\left(3 y_{2}-30 h y_{4}\right) x_{3}-\left(y_{1}-30 h y_{3}\right) x_{4}=0 .
$$

This shows us that the equations of the null system of the osculating cubic are

$$
\xi_{1}=y_{4}, \quad \xi_{2}=-3 y_{3}, \quad \xi_{3}=3 y_{2}-30 h y_{4}, \quad \xi_{4}=-y_{1}+30 h y_{3},
$$

where $\xi_{1}, \cdots, \xi_{4}$ are now the coordinates of the plane corresponding to the point with the coordinates $y$. From these equations it is easy to derive the result that the coordinates of the consecutive principal point referred to the original system are

$$
21 a h, 1+7 c h, 2 h, 0 \text {. }
$$

There is one more vertex of the consecutive tetrahedron of reference whose coordinates relative to the $x y z$-system are to be determined. The polar line of the point $(21 a h, 1+7 c h, 2 h, 0)$ with respect to the conic given by equation (16) has the equation

$$
h x_{1}-x_{2}+14 a h x_{3}=0 .
$$

Solution of this equation with (16) gives

$$
12 h, 14 a h, 1,3 h \text {, }
$$

as the coordinates referred to the original system of the point distinct from the point $Q$, in which the polar line of the consecutive principal point with respect to the consecutive osculating conic meets the conic. This is the required vertex.

Finally, in order to determine the equations of transformation between the original coordinate system and the consecutive one, it is sufficient, with the information we already have, to know the coordinates in the first system of the unit point. The plane determined by the consecutive point of Sannia, the consecutive principal point, and the point distinct from the point $Q$ in which the polar line of the consecutive principal point with respect to the consecutive osculating conic meets the conic is found to be

$$
x_{1}-21 a h x_{2}-9 h x_{3}-(1+7 c h) x_{4}=0 \text {, }
$$


or in non-homogeneous coordinates,

$$
1-21 a h x-9 h y-(1+7 c h) z=0 .
$$

Solution of this equation with equations (15) for the consecutive osculating cubic is made simple by assuming the value of $x$ to be of the form $1+r h$, an assumption which is permissible since we already know the required solution has $x=1$ for the case $h=0$. When the result is expressed in homogeneous coordinates we have for the consecutive unit point referred to the original coordinate system the point

$[1,1-h(5+7 a+7 c / 3), 1-h(10+14 a+14 c / 3), 1-h(9+21 a+7 c)]$.

We are now ready to derive the equations of transformation between the coordinates $x_{1}, x_{2}, x_{3}, x_{4}$ in the original system and the coordinates, which we shall denote by $X_{1}, X_{2}, X_{3}, X_{4}$, in the consecutive system, since we have found the coordinates, in each of the two systems, of five points no four of which are coplanar, namely, the four vertices of the consecutive tetrahedron and its unit point. We write the general linear equations of transformation of the coordinates $x$ into the coordinates $X$. Substitution in these equations of the coordinates of each of the five pairs of corresponding points yields twenty equations homogeneous in the sixteen constants of the transformation and in five proportionality factors. When the coefficients of the transformation are determined from these equations, we obtain, after simplification, the following result:

The equations of transformation from the original system to the consecutive system are

$$
\begin{array}{lrrr}
\rho x_{1}= & X_{1}+ & 21 a h X_{2}+ & 12 h X_{3}, \\
\rho x_{2}=h X_{1}+(1-7 c h / 3) X_{2}+ & 14 a h X_{3}+ & 6 h X_{4}, \\
\rho x_{3}= & 2 h X_{2}+(1-14 c h / 3) X_{3}+ & 7 a h X_{4}, \\
\rho x_{4}= & 3 h X_{3}+(1-7 c h) X_{4},
\end{array}
$$

where $\rho$ is a proportionality factor.

In non-homogeneous coordinates they become

$$
\begin{aligned}
& x=X+h\left(1-7 c X / 3+14 a Y+6 Z-21 a X^{2}-12 X Y\right) \\
& y=Y+h\left(2 X-14 c Y / 3+7 a Z-21 a X Y-12 Y^{2}\right) \\
& z=Z+h(3 Y-7 c Z-21 a X Z-12 Y Z)
\end{aligned}
$$

By interchanging $x_{1}, x_{2}, x_{3}, x_{4}$ with $X_{1}, X_{2}, X_{3}, X_{4}$, respectively, in equations (23), and changing the sign of $h$, the inverse transformation in homogeneous coordinates is readily found to be given by the equations 


$$
\begin{array}{lrrr}
\sigma X_{1} & =x_{1}- & 21 a h x_{2}- & 12 h x_{3}, \\
\sigma X_{2} & -h x_{1}+(1+7 c h / 3) x_{2}- & 14 a h x_{3} & 6 h x_{1}, \\
\sigma X_{3} & -2 h x_{2}+(1+14 c h / 3) x_{3}- & 7 a h x_{4}, \\
\sigma X_{4}= & & -3 h x_{3}+(1+7 c h) x_{4},
\end{array}
$$

where $\sigma$ is a factor of proportionality:

It is now possible to determine the equations of the curve $C$ referred to the consecutive coordinate system. In equations (2) we substitute for the variables $x, y, z$ their values in terms of $X, Y, Z$ as given by equations (24). In the resulting equations we replace $Y$ and $Z$ by power series in $X$ with undetermined coefficients. Since we now have two identities in $X$, the coefficient of each power of $X$ can be equated to zero. Solution of the equations so obtained, for the coefficients of the power series representing $Y$ and $Z$, permits us to write for the equations of $C$ in the coordinates $X, Y, Z$,

$$
Y=X^{2}+A X^{7}+B X^{8}+\cdots, Z=X^{3}+X^{6}+C X^{7}+D X^{8}+\cdots,
$$

where $A, B, C, D$ are defined by

$$
\begin{aligned}
& A=a+h(12+8 b-56 a c / 3), \\
& B=b+h(12 c-7 a d-14 b c+9 e), \\
& C=c+h\left(8 d-24 a-28 c^{2} / 3\right), \\
& D=d+h(9 g-28 a c-6-35 c d / 3-3 b) .
\end{aligned}
$$

By means of transformation (25) and the relations (26) we can now find the equation, or equations, of the locus consecutive to a given locus, not only from the geometric definition of the locus, as we have been doing throughout this section, but also by a more direct method. We write equations identical with the homogeneous form of those of the given locus, except that $x_{1}, x_{2}, x_{3}, x_{4}$ are replaced by $X_{1}, X_{2}, X_{3}, X_{4}$, and $a, b, c, d, \cdots$ by $A, B, C, D, \cdots$, and then apply transformations (25) and (26). It is obvious that the method to be used to obtain the point consecutive to one whose coordinates are known, is to substitute the given coordinates in equations (23).

\section{Applications of the transformation}

$\S I V$ is concerned with some applications which can be made of the results obtained in the preceding section. The problems we shall consider are of three types. The first of these is the determination of the tangents of the loci of various covariant points associated with a point $P$ on the curve $C$. We have already found the consecutive points to a number of covariant points, as, for example, the vertices of the original tetrahedron of reference. Each covariant 
point with its consecutive point determines a line which is the tangent at the point to its locus as the point $P$ moves along $C$. Such covariant curves as intersect their consecutive curves have envelopes generated by the intersection points. The envelopes are the edges of regression of the surfaces generated by the curves, and the intersection points are the focal points on the edges of regression. Our second problem is to find out which covariant curves that we have discussed have envelopes and to determine their contact or focal points. In the third kind of problem we determine the characteristic curve of a covariant surface, that is, the curve of intersection of a surface with its consecutive surface. We go yet farther than this and find the focal point of the edge of regression of the envelope of the surface, that is, the point in which a characteristic curve intersects its consecutive curve.

We turn now to the determination of the tangents of the loci of some covariant points. Obviously, the locus of the point $(1,0,0,0)$ is the curve $C$ itself and its tangent at any point is the tangent to $C$ at that point. The points consecutive to the other three vertices

$$
(0,1,0,0), \quad(0,0,1,0),(0,0,0,1)
$$

of the tetrahedron of reference are, respectively,

$$
(21 a h, 1+7 c h, 2 h, 0), \quad(12 h, 14 a h, 1,3 h), \quad(0,6 h, 7 a h, 1) .
$$

Hence we find for the tangents to the respective loci of the points $(0,1,0,0)$, $(0,0,1,0),(0,0,0,1)$, the lines whose equations are

$$
\begin{aligned}
2 x_{1}-21 a x_{3} & =x_{4}=0, \\
7 a x_{1}-6 x_{2} & =x_{1}-4 x_{4}=0, \\
7 a x_{2}-6 x_{3} & =x_{1}=0 .
\end{aligned}
$$

The point

$$
[1,1-h(5+7 a+7 c / 3), 1-h(10+14 a+14 c / 3), 1-h(9+21 a+7 c)]
$$

is consecutive to the unit point $(1,1,1,1)$; together these points determine, as tangent of the locus of the unit point, the line

$$
x_{1}-2 x_{2}+x_{3}=(2 R-6) x_{1}-(3 R-6) x_{2}+R x_{4}=0,
$$

where $R$ is defined by

$$
R=5+7 a+7 c / 3 .
$$

The point consecutive to the point of Sannia $(1,0,0,1)$ is the point $(1,7 \mathrm{~h}$, $7 a h, 1-7 c h)$. This gives us that the tangent to the locus of the point $(1,0,0,1)$ is the line 


$$
a x_{2}-x_{3}=a x_{1}-c x_{3}-a x_{4}=0 .
$$

As an example of the second type of problem we consider first the case of the osculating conic, whose equations are given by (4). Solution of these equations with equations (16) of the consecutive osculating conic gives the point $(1,0,0,0)$ for the only intersection of the two curves. Therefore, the osculating conic has no envelope but the curve $C$ itself. The case of the osculating cubic proves more fruitful. If we make equations (3) and (15) homogeneous and solve them simultaneously we find that the contact points of the osculating cubic with its envelope are the points $(1,0,0,0)$ and $(0,0,0,1)$.

We now investigate the situation for some covariant lines. It is obvious that the envelope of the tangent line is the curve $C$. Let us consider some other edge of the tetrahedron of reference, as the line $x_{1}=x_{2}=0$. Its consecutive line is $X_{1}=X_{2}=0$, or by applying (25),

$$
x_{1}-21 a h x_{2}-12 h x_{3}=-h x_{1}+(1+7 c h / 3) x_{2}-14 a h x_{3}-6 h x_{4}=0 .
$$

There is no common solution of these four equations, and hence the line $x_{1}=x_{2}=0$ does not intersect its consecutive line. In a similar way we find that the other edges of the tetrahedron of reference are skew to the corresponding edges of the consecutive tetrahedron. There are many other covariant lines we might consider, for example, the lines joining the point of Sannia to the vertices $(0,1,0,0)$ and $(0,0,1,0)$, and to the unit point, and the lines joining the unit point to the vertices of the tetrahedron. In each case we write the equations of the line determined by the two points considered, then write identical equations only with coordinates $X$ instead of coordinates $x$ and apply transformation (25). In every instance we find that the four equations so obtained have no common solution. We may sum up these findings as follows:

The tangent line has the curve $C$ for envelope, but the other edges of the tetrahedron of reference, the lines joining the point of Sannia or the unit point to a vertex of the tetrahedron, and the line joining the point of Sannia with the unit point do not generate developable surfaces and hence have no envelopes.

Our third kind of problem is concerned with the characteristic curves and edges of regression of the envelopes of covariant surfaces. Let us take first the plane $x_{1}=0$. The equation of the consecutive plane is $X_{1}=0$ or, after making use of (25),

$$
x_{1}-21 a h x_{2}-12 h x_{3}=0 .
$$

The characteristic line of the plane $x_{1}=0$ is then the intersection of these two planes, which is the same as the intersection of the two planes

$$
x_{1}=0, \quad 7 a x_{2}+4 x_{3}=0 .
$$


To find the point at which the plane $x_{1}=0$ touches the edge of regression we must know the consecutive characteristic line. Since the characteristic line is a covariant configuration and is determined by the two planes (27), its consecutive characteristic line is determined by the two consecutive planes

$$
X_{1}=0,7 A X_{2}+4 X_{3}=0 .
$$

The first of these gives us nothing new, but when the second has been subjected to transformations (25) and (26) we find that the point where the plane $x_{1}=0$ touches the edge of regression is determined by the system of planes with the equations

$$
\begin{gathered}
x_{1}=0, \quad 7 a x_{2}+4 x_{3}=0, \\
(76+56 b-147 a c) x_{2}-98 a^{2} x_{3}-70 a x_{4}=0 .
\end{gathered}
$$

After solving these equations, we state our conclusions: the characteristic line of the plane $x_{1}=0$ has the equations (27); the focal point of the edge of regression of the developable of this plane has the coordinates

$$
0,140 a,-245 a^{2}, M \text {, }
$$

where $M$ is defined by

$$
M=152+112 b-294 a c+343 a^{3} .
$$

In a similar way we find the characteristic lines and points of contact with the edges of regression of the developables of other covariant planes. The characteristic lines of the planes $x_{2}=0, x_{3}=0, x_{4}=0$, and of the plane $x_{1}-x_{4}=0$ determined by the point of Sannia and the two vertices $(0,0,1,0)$ and $(0,1,0,0)$ have, respectively, the equations

$$
\begin{aligned}
x_{2}=x_{1}+14 a x_{3}+6 x_{4} & =0, \quad x_{3}=2 x_{2}+7 a x_{4}=0, \quad x_{8}=x_{4}=0, \\
x_{1}-x_{4} & =21 a x_{2}+9 x_{3}+7 c x_{4}=0 .
\end{aligned}
$$

The respective focal points of the developables of these planes are

$$
\begin{gathered}
\left(3 M-42-343 a^{3}, 0,21 c-49 a^{2}, N\right), \\
(3-N,-7 a, 0,2), \quad(1,0,0,0), \\
\left(3 M-S, 49 c^{2}-343 a^{2} c-84 d+567 a, 196 a d-1323 a^{2}-273 c\right. \\
\left.-196 b c+343 a^{2}, 3 M-S\right),
\end{gathered}
$$

where $M$ is as defined in equation (28) and $N$ and $S$ are given by

$$
N=98 a c-56 b-69, S=105+84 b-294 a c .
$$

The characteristic curve of the osculating quadric cone is the curve of 
intersection of the two quadric surfaces whose non-homogeneous equations are (6) and (19). This is equivalent to the curve of intersection of the first surface and the surface with homogeneous equation

$$
x_{2} x_{3}-x_{1} x_{4}-6 x_{4}^{2}=0 ;
$$

it is composed of the tangent line $x_{3}=x_{4}=0$ and the cubic curve

$$
x_{1}=t^{3}-6, \quad x_{2}=t^{2}, \quad x_{3}=t, \quad x_{4}=1 .
$$

Hence, we state the conclusion: the characteristic curve of the osculating quadric cone of the curve $C$ at the point $P$ consists of the tangent line to the curve $C$ at the point $P$ and the cubic (29). This cubic has no contact with the curve $C$ at the point $P$. The cone has seven-point contact with its edge of regression at $(1,0,0,0)$ and touches it again at the point

$$
\left(343 c^{3}-750,245 c^{2}, 175 c, 125\right) .
$$

From equations (7) and (21) made homogeneous we find that the intersection of the two quadric surfaces

$$
x_{1} x_{3}-x_{2}^{2}=0, \quad 12\left(x_{2} x_{4}-x_{3}^{2}\right)-7 a\left(x_{1} x_{4}-x_{2} x_{3}\right)=0
$$

forms the characteristic of the seven-point cone with vertex at the Halphen point. As we should expect since this cone also contains the osculating cubic, the osculating cubic makes up a part of the characteristic, the remainder being the line whose equations are

$$
7 a x_{1}-12 x_{2}=0, \quad 7 a x_{2}-12 x_{3}=0 .
$$

So we state the following result:

The characteristic of the seven-point cone with vertex at the Halphen point corresponding to the point $P$ of the curve $C$ consists of the osculating cubic to $C$ at $P$ and of the line with equations (30). This cone has five-point contact with its edge of regression at $(1,0,0,0)$, iwo-point contact at

$$
\left(1728,1008 a, 588 a^{2}, 343 a^{3}\right) \text {, }
$$

and single contact at

$$
\left(1728,1008 a, 588 a^{2}, 1372 a^{3}-24 T\right),
$$

where $T$ is defined by

$$
T=96+56 b-147 a c .
$$

The characteristic curve of the surface of Calapso whose equation is 
given by ( 8 ) is the intersection of the two cubic surfaces

$$
\begin{gathered}
x_{1} x_{4}^{2}-3 x_{2} x_{3} x_{4}+2 x_{3}^{8}=0, \\
2 x_{2}^{2} x_{4}-x_{2} x_{3}^{2}+2 x_{3} x_{4}^{2}-x_{1} x_{3} x_{4}=0 ;
\end{gathered}
$$

it, also, includes the tangent line $x_{3}=x_{4}=0$ as a part.

A second procedure for determining characteristic curves is suggested by the fact, which we recall from differential geometry, that the characteristic curve of a surface belonging to a one-parameter family has for its equations the equation of the surface and the derivative of that equation with respect to the parameter. To use this method we see at once that we need differentiation formulas for $x_{1}, x_{2}, x_{3}, x_{4}$ with respect to the parameter $h$. These are deduced from equations (25) in which $\sigma$ is taken to be 1 . In each equation the term on the right which is free of $h$ is transposed to the left; then both members of the equation are divided by $h$. Taking the limits as $h$ approaches 0 , we obtain the formulas of differentiation for $x_{1}, x_{2}, x_{3}, x_{4}$ with respect to $h$ :

$$
\begin{aligned}
& x_{1}^{\prime}=-21 a x_{2}-12 x_{3}, \\
& x_{2}^{\prime}=-x_{1}+7 c x_{2} / 3-14 a x_{3}-6 x_{4}, \\
& x_{3}^{\prime}=\quad-2 x_{2}+14 c x_{3} / 3-7 a x_{4}, \\
& x_{4}^{\prime}=\quad-3 x_{3}+7 c x_{4},
\end{aligned}
$$

where $x_{1}^{\prime}$ denotes the derivative of $x_{1}$ with respect to $h$, and so on. As an instance of the application of these formulas to the solution of our problem we shall consider the osculating quadric cone with equation

$$
x_{3}^{2}-x_{2} x_{4}=0 .
$$

Differentiation of this equation gives

$$
2 x_{8} x_{3}^{\prime}-x_{2} x_{4}^{\prime}-x_{2}^{\prime} x_{4}=0 .
$$

Substitution from (31), followed by simplification by means of the equation of the osculating quadric cone, corroborates our conclusion of a previous paragraph, that the characteristic curve of this quadric is its intersection with the quadric $x_{1} x_{4}-x_{2} x_{3}+6 x_{4}^{2}=0$.

Finally, we mention the cross ratio of four lines in the plane $x_{1}=0$ through the Halphen point $(0,0,0,1)$. The two edges of the tetrahedron of reference

$$
x_{1}=x_{2}=0, \quad x_{1}=x_{3}=0,
$$

the tangent to the locus of the point $(0,0,0,1)$ with equations 
A. V. NEWTON

$$
x_{1}=7 a x_{2}-6 x_{3}=0 \text {, }
$$

and the characteristic line of the plane $x_{1}=0$ with equations

$$
x_{1}=7 a x_{2}+4 x_{8}=0 \text {, }
$$

have cross ratio equal to $-3 / 2$.

\section{University of Chicago,}

$$
\text { Chicago, Irl. }
$$

